\title{
The interaction between HIV testing social norms and self-efficacy on HIV testing among Chinese men who have sex with men: results from an online cross-sectional study
}

Peizhen Zhao ${ }^{1+}$, Li Liu ${ }^{2+}$, Ye Zhang ${ }^{1}$, Huanhuan Cheng ${ }^{3}$, Bolin Cao ${ }^{4,5}$, Chuncheng Liu ${ }^{4}$, Cheng Wang ${ }^{1}$, Bin Yang ${ }^{1}$, Chongyi Wei ${ }^{6}$, Joseph D. Tucker ${ }^{4,5,7}$ and Weiming Tang ${ }^{1,4,5,7^{*}}$ (D)

\begin{abstract}
Background: Increasing human immunodeficiency virus (HIV) testing is critical for HIV control. This study aimed to evaluate the interaction between social norms and self-efficacy on HIV testing among Chinese men who have sex with men (MSM).

Methods: We conducted an online survey in eight Chinese cities in Shandong and Guangdong Provinces in July 2016. We included participants who were born as a male, at least 16 years old, currently living in one of the designated cities, and had ever engaged in anal sex with a man. We collected information regarding sociodemographics, high-risk behaviors, and history of HIV and other STI testing. We coded sensitivity to social norms using six items asking participants about their perceived social norm regarding HIV testing. We coded HIV testing self-efficacy using a separate six-item scale. We interpreted higher mean scores as higher sensitivity to social norms and higher self-efficacy, respectively. We conducted logistic regressions to evaluate the interaction between selfefficacy and social norms on HIV testing.

Results: A total of 2105 men completed the survey. The mean age of the participants was $25.97 \pm 6.42$ years. Over four-fifths (85.9\%) of participants were unmarried, $22.7 \%$ were students, and $64.6 \%$ at least had a college degree. 62.5 and $32.6 \%$ of participants ever and tested HIV in the last three months, respectively.

With respect to uptake of HIV testing in the last three months, the adjusted odds ratio was 1.01(95\% Cl: 0.96-1.06) for higher sensitivity to social norms and 1.09 (95\% Cl: 1.05-1.14) for higher self-efficacy, with an interaction effect of 1.02 (95\% Cl: 1.01-1.03), respectively. With respect to uptake of lifetime HIV testing, the adjusted odds ratio was 1.03(95\% Cl: 0.99-1.07) for higher sensitivity to social norms and 1.15 (95\% Cl: 1.11-1.19) for higher self-efficacy, with an interaction effect of 1.02 (95\% Cl: 1.01-1.04), respectively.

Conclusions: Our survey demonstrated that there is a significant association between the uptake of HIV testing with sensitivity to the social norm, higher self-efficacy, as well as the interaction between them. Tailored studies for improving HIV testing among MSM in China can combine these two interventions together.
\end{abstract}

Keywords: Men who have sex with men (MSM), Social norm, Self-efficacy, HIV testing

\footnotetext{
* Correspondence: weimingtangscience@gmail.com

${ }^{\dagger}$ Peizhen Zhao and Li Liu contributed equally to this work.

Peizhen Zhao and Li Liu are co-first authors to this work.

'Dermatology Hospital, Southern Medical University, Guangzhou, China

${ }^{4}$ SESH study group of University of North Carolina at Chapel Hill, Guangzhou,

China

Full list of author information is available at the end of the article
}

(c) The Author(s). 2018 Open Access This article is distributed under the terms of the Creative Commons Attribution 4.0 International License (http://creativecommons.org/licenses/by/4.0/), which permits unrestricted use, distribution, and reproduction in any medium, provided you give appropriate credit to the original author(s) and the source, provide a link to the Creative Commons license, and indicate if changes were made. The Creative Commons Public Domain Dedication waiver (http://creativecommons.org/publicdomain/zero/1.0/) applies to the data made available in this article, unless otherwise stated. 


\section{Introduction}

Men who have sex with men have become a key population for HIV infection [1]. Increasing the uptake of HIV testing among MSM is a crucial component of the HIV treatment cascade and the control of the global HIV epidemic [2].

To scale up HIV testing services, the Chinese government has established voluntary counseling and testing (VCT) clinics that offer free HIV testing services and provider-initiated testing and counseling (PITC) [3], but only $60 \%$ of Chinese men who have sex with men (MSM) have ever tested for HIV [4]. Among Chinese MSM, potential reasons for low uptake of HIV testing include stigma against HIV, limited access to HIV testing service, and poor sexual orientation disclosure $[3,5,6]$. Identifying potential strategies to reduce these barriers and further improving HIV testing uptake among Chinese MSM is essential.

Studies in China and other countries indicated that HIV testing social norm and self-efficacy are two important psychosocial factors can facilitate HIV testing among MSM [7-9]. Self-efficacy refers to the belief in one's own ability to complete tasks and reach goals [8]. According to the social cognitive theory, self-efficacy plays an important role in the adoption, initiation, and maintenance of health behaviors [8]. HIV testing self-efficacy refers to people's level of confidence to have HIV testing [10]. Social norms are social attitudes of approval or disapproval that specify what ought and ought not to be done and are significant in the context of health [11]. HIV testing social norms refer to people's social attitudes about HIV testing [11]. Improving HIV testing self-efficacy and perceived positive social norms of HIV testing are two intervention strategies for improving uptake of HIV testing among MSM $[8,9,12]$.

Even HIV testing social norm and self-efficacy has a positive impact on promoting HIV testing, whether these two can interact with each other and further strengthen the intervention effect is still not clear while knowing this is critical for designing tailored interventions for MSM. Therefore, the aim of this study was to examine whether there exists an interaction effect between perceived HIV testing-related social norm and self-efficacy on HIV testing among Chinese MSM. The hypothesis of this study is that the perceived HIV testing-related social norm and self-efficacy can interact with each other and further improve HIV testing uptake.

\section{Methods}

\section{Study population}

A nationwide cross-sectional online survey among MSM was conducted in eight Chinese cities: Guangzhou, Shenzhen, Zhuhai, Jiangmen (Guangdong Province, Southern China), Jinan, Qingdao, Jining, and Yantai (Shandong Province, Northern China) in July 2016. These eight cities of Guangdong and Shandong Province were chosen because they were urban cities with the relatively high prevalence of HIV.

We recruited participants online by collaborating with a gay dating application. Banner advertisements with links to the online survey were sent to registered users of the application. Viewers who clicked the survey link were directed to the web survey.

We only included participants who were born biologically as a male, at least 16 years old, and currently living in one of the designated cities who had had anal sex with a man at least once during their lifetime. We also required participants to provide their cell phone numbers for other follow-up purposes and agree to informed consent. All eligible participants received a small phone card reimbursement or WeChat incentive transfer (equivalent to roughly 7.5 USD).

\section{Measures}

After screening for eligibility and signing the informed consent, we first collected information on socio-demographic information, including age (which we further categorized into three groups: less than 20, 20-29, or 30 and above), marital status (never married or ever married, including widowed or divorced), occupation (student or not), education (senior high school or below, college/bachelors, or masters or $\mathrm{PhD}$ ), and monthly income (less than $250 \mathrm{USD}$, 250-500 USD, 501-800 USD, 801-1250 USD or above 1250 USD). We also asked participants whether they had ever been tested for HIV (including HIV self-testing and facility-based testing) in their lifetime, whether ever self-tested, and whether testing for HIV in the last 3 months. HIV testing in lifetime was measured by asking participants whether they performed any HIV testing in their lifetime, including testing in general hospitals, clinics, the point of care sites or HIV self-testing. The HIV self-testing was defined as people tested HIV in private and interpreted the results on their own [13]. HIV testing in the past 3 months was measured as whether the participants performed any HIV testing in the last 3 months.

\section{HIV testing self-efficacy}

HIV testing self-efficacy was measured using six questions that elicited participants' attitudes toward and confidence in receiving HIV testing $[14,15]$. The items are shown in Table 2. All items were scored using a 4-point Likert-type response set, with answers ranging from strongly agree (4) to strongly disagree (1). For each participant, a mean score was calculated with 4 as the highest possible score of 4 and 1 as the possible lowest score. Higher mean scores indicated higher HIV testing self-efficacy. The scale assessing respondents' overall levels of HIV testing self-efficacy was found to be reliable (Cronbach's $\alpha=0$.792). 


\section{HIV testing social norm}

Social norm was measured by six items asking participants about their awareness of social norms about HIV testing $[11,16]$ (see Table 2). All items were scored using a 4-point Likert-type response set, with answers ranging from strongly agree (4) to strongly disagree (1). For each participant, a mean score was calculated with 4 as the highest possible score of 4 and 1 as the possible lowest score. Higher mean scores indicated higher sensitivity to HIV testing social norms. The Cronbach's $\alpha$ of HIV testing social norm was 0.695 .

\section{Statistical analysis}

We used descriptive analysis to describe socio-demographics, high-risk behaviors, and self-efficacy among the participants who had been tested for HIV in the past three months and those who had not. We also analyzed the linear assumption through semiparametric regression map (Additional file 1). Spearmen correlation tests were used to identify any association between social norm and self-efficacy. Univariate and multivariable logistic regressions were used to evaluate the association between HIV testing and self-efficacy and HIV testing and sensitivity to social norms. We used a logistic regression multiplication model to analyze the association of the interaction between HIV testing social norm and HIV testing self-efficacy. We used multivariable logistic regression to analyze the influencing factors of HIV testing. Age, education, marital status, and income were a covariate in the model. These variables were chosen based on our prior knowledge, and a directed acyclic graph (DAG) [17] was drawn to assist in this analysis. Throughout all the analyses, results are reported as statistically significant whenever $P<0.05$. All data were analyzed using SAS 9.4 (SAS Int. Cary, NC, USA).

\section{Results}

\section{Socio-demographic characteristics and behaviors}

Two thousand one hundred five participants finished the online survey. The mean age of participants was $26.0 \pm$ 6.4 years. $54.4 \%$ of the participants were $\leq 25$ years old, $14.1 \%$ were married, $22.7 \%$ were students, $64.6 \%$ had a college or above degree, and $71.6 \%$ had an annual income less than $\$ 9700$ USD (60,000 RMB). Also, $72.4 \%$ of the participants self-identified as gay, and $23.6 \%$ self-identified as bisexual (Table 1).

\section{HIV testing}

Among 2105 participating MSM, 1315 (62.5\%) reported being tested for HIV at least once in their lifetime. However, only $687(32.6 \%)$ participants had been tested for HIV within the past 3 months. Moreover, 685(32.5\%) participants reported having self-tested for HIV at least once in their lifetime.
Table 1 Sociodemographic characteristics of MSM participants $(n=2105)$

\begin{tabular}{|c|c|c|}
\hline Characteristics & Frequency $(N=2105)$ & Percentage (\%) \\
\hline \multicolumn{3}{|l|}{$\mathrm{Age}^{\mathrm{a}}$} \\
\hline$\leq 25$ & 1146 & 54.44 \\
\hline $26-35$ & 789 & 37.48 \\
\hline $36-45$ & 149 & 7.08 \\
\hline$\geq 46$ & 21 & 1.00 \\
\hline \multicolumn{3}{|l|}{ Student } \\
\hline Yes & 477 & 22.66 \\
\hline No & 1628 & 77.34 \\
\hline \multicolumn{3}{|l|}{ Marital status } \\
\hline Not married & 1809 & 85.94 \\
\hline Engaged or Married & 187 & 8.88 \\
\hline Separated or Divorced & 106 & 5.04 \\
\hline Widowed & 3 & 0.14 \\
\hline \multicolumn{3}{|l|}{ Education level } \\
\hline High school or below & 746 & 35.44 \\
\hline College diploma & 583 & 27.70 \\
\hline Undergraduate & 697 & 33.11 \\
\hline Postgraduate (Master/PhD) & 79 & 3.75 \\
\hline \multicolumn{3}{|l|}{ Individual monthly income } \\
\hline$<1500 \mathrm{RMB}$ & 391 & 18.57 \\
\hline 1500-3000 RMB & 425 & 20.19 \\
\hline 3001-5000 RMB & 690 & 32.78 \\
\hline 5001-8000 RMB & 384 & 18.24 \\
\hline$>8000 \mathrm{RMB}$ & 215 & 10.21 \\
\hline \multicolumn{3}{|l|}{ Gender identity } \\
\hline Male & 1999 & 94.96 \\
\hline Female & 33 & 1.57 \\
\hline Transgender & 34 & 1.62 \\
\hline Unsure/Other & 39 & 1.85 \\
\hline \multicolumn{3}{|l|}{ Sexual orientation } \\
\hline Homosexual & 1524 & 72.40 \\
\hline Bisexual & 496 & 23.56 \\
\hline Heterosexual & 11 & 0.52 \\
\hline Unsure/Other & 74 & 3.52 \\
\hline \multicolumn{3}{|l|}{ HIV testing in lifetime } \\
\hline Yes & 1315 & 62.5 \\
\hline No & 790 & 37.5 \\
\hline \multicolumn{3}{|l|}{ HIV Testing in the past 3 months } \\
\hline Yes & 687 & 32.6 \\
\hline No & 1418 & 67.4 \\
\hline \multicolumn{3}{|l|}{ HIV self-testing in lifetime } \\
\hline Yes & 685 & 32.5 \\
\hline No & 1420 & 67.5 \\
\hline
\end{tabular}

${ }^{\mathrm{a}}$ Age: mean $=25.97, \mathrm{SD}= \pm 6.42$ 


\section{Social norm and self-efficacy}

In this study, the median(interquartile range, IQR) score of HIV testing social norms was 17.0 (15.0-18.0). While around $75 \%$ of participants endorsed all the statements of HIV testing social norms, between 17.2 and $52.2 \%$ of participants did not endorse each individual item. For HIV testing self-efficacy, the median (IQR) score was 19.0 (17.0-21.0). A majority of participants endorsed these statements, while a small proportion $(2.8-26.8 \%)$ did not endorse individual items. (Table 2).

\section{The association between HIV testing with sensitivity to social norms and self-efficacy}

Univariate analysis indicated that MSM with higher sensitivity to social norms had a higher proportion of HIV testing in the past 3 months, though the result was not significant (Crude odds ratio (cOR, main effect) $=1.02$ [0.96-1.06]). Univariate analysis also indicated that MSM with higher self-efficacy had more HIV testing in the past 3 months, with a cOR (main effect) of 1.09 [1.05-1.13]. We also detected an association of the interaction between sensitivity to HIV testing social norms and self-efficacy with HIV testing in the last 3 months $(\mathrm{cOR}=1.02$ [1.01,1.04], see Table 3).

Furthermore, univariate analysis indicated that MSM with higher sensitivity to the social norm was more likely to have had HIV testing at least once in their lifetime, though the difference was not significant $(\mathrm{cOR}=1.02$, [0.98-1.06]). Univariate analysis also revealed that MSM with higher self-efficacy score was more likely to have had HIV testing at least once in their lifetime $(\mathrm{cOR}=1.09[0.96-1.06])$. We also detected an association of the interaction between sensitivity to HIV testing social norms and self-efficacy with lifetime HIV testing $(\mathrm{cOR}=1.02[1.01,1.04]$, see Table 3$)$.

Univariate analysis demonstrated that MSM with higher sensitivity to social norms had higher lifetime HIV self-testing in a lifetime ( $\mathrm{cOR}=1.12[1.07,1.18]$ ). MSM with higher self-efficacy score was more likely to have lifetime HIV self-testing in a lifetime, though the effect was not significant ( $\mathrm{cOR}=1.03$, [0.99-1.07], see Table 3). Similar results were observed after adjusting for potential confounders (age, marital status, education level, and monthly income).

In our multivariate analysis, MSM with higher self-efficacy was more likely to have HIV testing in the past 3 months (adjusted odds ratio, aOR $=1.09[1.05,1.14]$ ). For the increasing of every additional self-efficacy score, the HIV testing proportion in the past 3 months increased by 1.09 folds. Further, the interaction between social norm and self-efficacy was also associated with HIV testing in the past 3 months $(\mathrm{aOR}=1.02[1.01,1.03])$. MSM with higher self-efficacy and higher social norm were more likely to have HIV testing in the past 3 months.

In our multivariate analysis, MSM with higher self-efficacy and higher sensitivity to social norms had higher lifetime HIV testing proportion $(\mathrm{aOR}=1.15[1.11,1.19]$ and 1.12 $[1.06,1.78]$, respectively). For every additional increase of self-efficacy score, the HIV testing proportion in the lifetime increased by 1.15 folds. For every additional increase of social norms score, the HIV testing proportion in the lifetime increased by 1.12 folds. Further, the interaction effect between social norm and self-efficacy was also associated with lifetime HIV testing $(\mathrm{aOR}=1.02[1.01,1.04]$,

Table 2 Distribution of responses for items related to social norms and self-efficacy

\begin{tabular}{|c|c|c|c|c|}
\hline & Strongly agree & Agree & Disagree & $\begin{array}{l}\text { Strongly } \\
\text { disagree }\end{array}$ \\
\hline \multicolumn{5}{|l|}{ Social Norm } \\
\hline Most gay men want to get tested but are afraid to get tested. & $513(24.37)$ & $977(46.41)$ & $517(24.56)$ & $98(4.66)$ \\
\hline Most gay men who get tested do not want others to find out they were tested. & $746(35.44)$ & $969(46.03)$ & $341(16.20)$ & $49(2.33)$ \\
\hline Most gay men want to get tested for HIV. & $762(36.20)$ & $981(46.60)$ & $345(16.39)$ & $17(0.81)$ \\
\hline Most gay men who want to get tested will tell their partners they want to get tested & $490(23.28)$ & $995(47.27)$ & $575(27.32)$ & $45(2.14)$ \\
\hline Most gay men have been tested for HIV & $323(15.34)$ & $684(32.49)$ & $968(45.99)$ & $130(6.18)$ \\
\hline Most gay men get tested for HIV only if they are sick or feel uncomfortable & 393(18.67) & $968(45.99)$ & $634(30.12)$ & $110(5.23)$ \\
\hline \multicolumn{5}{|l|}{ Self-Efficacy } \\
\hline You would feel comfortable discussing HIV testing with a potential partner & $508(24.13)$ & 1033(49.07) & 495(23.52) & $69(3.28)$ \\
\hline $\begin{array}{l}\text { You feel confident that you could refuse to have sex with a partner who did not } \\
\text { want to undergo HIV testing }\end{array}$ & $686(32.59)$ & $860(40.86)$ & $447(21.24)$ & 112(5.32) \\
\hline You feel confident that you could persuade your partner to undergo HIV testing & $717(34.06)$ & $1152(54.73)$ & 224(10.64) & $12(0.57)$ \\
\hline You can get tested for HIV if you wish & $1004(47.70)$ & $1043(49.55)$ & $52(2.47)$ & $6(0.29)$ \\
\hline You would get tested for HIV even if you are afraid to know the results & $925(43.94)$ & $1074(51.02)$ & $91(4.32)$ & $15(0.71)$ \\
\hline You have confidence that you will undergo HIV testing regularly & $541(25.70)$ & 1028(48.84) & $501(23.80)$ & $35(1.66)$ \\
\hline
\end{tabular}


Table 3 The association of HIV testing with Social Norms, Self-Efficacy, and their interaction

\begin{tabular}{|c|c|c|c|c|c|c|c|c|}
\hline & \multicolumn{4}{|c|}{ Crude Model } & \multicolumn{4}{|c|}{ Adjusted Model* } \\
\hline & Coefficient & SE & OR $(95 \% \mathrm{Cl})$ & $P$-value & Coefficient & SE & OR $(95 \% \mathrm{Cl})$ & $P$-value \\
\hline \multicolumn{9}{|c|}{ HIV Testing in the past 3 months } \\
\hline Intercept & -0.16 & 0.42 & - & - & 0.82 & 0.51 & - & - \\
\hline Social norm & 0.01 & 0.02 & $1.02(0.96,1.06)$ & 0.55 & 0.01 & 0.02 & $1.01(0.96,1.06)$ & 0.75 \\
\hline Intercept & -1.58 & 0.40 & - & - & -0.71 & 0.48 & - & - \\
\hline Self-efficacy & 0.09 & 0.02 & $1.09(1.05,1.13)$ & $<0.001^{\#}$ & 0.09 & 0.02 & $1.09(1.05,1.14)$ & $<0.001^{\#}$ \\
\hline Intercept & 5.10 & 2.88 & - & - & 5.47 & 2.92 & - & - \\
\hline Social norm & -0.40 & 0.17 & $0.67(0.48,0.93)$ & $0.018^{\#}$ & -0.38 & 0.17 & $0.69(0.49,0.96)$ & $0.029^{\#}$ \\
\hline Self-efficacy & -0.23 & 0.14 & $0.79(0.60,1.05)$ & 0.11 & -0.20 & 0.15 & $0.82(0.62,1.09)$ & 0.17 \\
\hline Social norm*Self-efficacy & 0.02 & 0.01 & $1.02(1.01,1.04)$ & $0.023^{\#}$ & 0.02 & 0.01 & $1.02(1.01,1.03)$ & $0.042^{\#}$ \\
\hline \multicolumn{9}{|l|}{ HIV testing in lifetime } \\
\hline Intercept & 0.24 & 0.35 & - & - & -2.20 & 0.43 & - & - \\
\hline Social norm & 0.02 & 0.02 & $1.02(0.98,1.06)$ & 0.42 & 0.03 & 0.02 & $1.03(0.99,1.07)$ & 0.20 \\
\hline Intercept & -2.07 & 0.31 & - & - & -4.42 & 0.41 & - & - \\
\hline Self-efficacy & 0.14 & 0.02 & $1.15(1,11,1.89)$ & $<0.001^{\#}$ & 0.14 & 0.02 & $1.15(1.11,1.19)$ & $<0.001^{\#}$ \\
\hline Intercept & & & & & -12.14 & 2.55 & - & - \\
\hline Social norm & 0.39 & 0.14 & $1.41(1.10,1.80)$ & $0.007^{\#}$ & 0.44 & 0.15 & $1.48(1.14,1.92)$ & $0.003^{\#}$ \\
\hline Self-efficacy & 0.54 & 0.12 & $1.64(1.32,2.04)$ & $<0.001^{\#}$ & 0.58 & 0.13 & $1.70(1.36,2.13)$ & $<0.001^{\#}$ \\
\hline Social norm*Self-efficacy & 0.02 & 0.01 & $1.02(1.01,1.04)$ & $0.001^{\#}$ & 0.03 & 0.01 & $1.02(1.01,1.04)$ & $0.0008^{\#}$ \\
\hline \multicolumn{9}{|l|}{ Self-HIV testing in lifetime } \\
\hline Intercept & -1.82 & 0.43 & - & - & -0.53 & 0.53 & - & - \\
\hline Social norm & 0.11 & 0.03 & $1.12(1.07,1.18)$ & $<0.001^{\#}$ & 0.11 & 0.03 & $1.12(1.06,1.18)$ & $<0.001^{\#}$ \\
\hline Intercept & -0.39 & 0.39 & - & - & 0.95 & 0.49 & - & - \\
\hline Self-efficacy & 0.02 & 0.02 & $1.03(0.99,1.07)$ & 0.22 & 0.02 & 0.02 & $1.02(0.99,1.07)$ & 0.24 \\
\hline Intercept & 2.09 & 2.81 & - & - & 3.29 & 2.87 & - & - \\
\hline Social norm & -0.04 & 0.17 & $0.91(0.68,1.22)$ & 0.54 & -0.04 & 0.17 & $0.92(0.68,1.23)$ & 0.56 \\
\hline Self-efficacy & -0.08 & 0.14 & $0.84(0.66,1.07)$ & 0.16 & -0.08 & 0.14 & $0.84(0.66,1.08)$ & 0.17 \\
\hline Social norm*Self-efficacy & 0.04 & 0.01 & $1.01(0.99,1.02)$ & 0.16 & 0.04 & 0.01 & $1.01(0.99,1.02)$ & 0.18 \\
\hline
\end{tabular}

*Model adjusted for age (Continuous), marital status (Not married, engaged or Married, separated or widowed), education level (High school or below, college or bachelors, or masters or $\mathrm{PhD}$ ) and monthly income ( $<1500 \mathrm{RMB}, 1500-3000 \mathrm{RMB}, 3001-5000 \mathrm{RMB}, 5001-8000 \mathrm{RMB}$ or $>8000 \mathrm{RMB}$ ). \# and bold indicates $P<0.05$

see Table 3). MSM with higher self-efficacy and higher social norm were more likely to have HIV testing in the life time.

\section{Discussion}

Previous studies have shown that HIV testing self-efficacy and social norms had an impact on the uptake of HIV testing. This study extends the existing literature by assessing the relationship between HIV testing and social norms, self-efficacy, and the potential interaction between social norms and self-efficacy.

Our study showed that sensitivity to HIV testing social norms was positively associated with having received an HIV testing within the past 3 months as well as lifetime HIV testing. This is consistent with the findings of previous studies from northern Nigeria and rural Uganda [7]. This finding supports the hypothesis that improving
HIV testing social norms can potentially improve the HIV testing uptake among Chinese MSM. Intervention packages aimed at improving HIV testing proportion should consider social norms and perceptions of social norms as an actionable part of the overall intervention strategies.

Our study also indicated that HIV testing self-efficacy was positively associated with lifetime HIV testing. This finding is consistent with previous studies, which have shown that improving HIV testing self-efficacy is useful for promoting HIV testing and medication adherence [18-20]. Our study suggests the importance of developing strategies (i.e. raising the severity perception [14]) to strengthen MSM self-efficacy, thereby improving safe sexual behavior and HIV testing proportion [21]. More importantly, integrating self-efficacy with other ongoing interventions (i.e., HIV self-testing), and further 
improving the effectiveness of these implementation strategies will be more practical.

Further, our study demonstrated that sensitivity to social norms and self-efficacy interact with each other in promoting HIV testing, and MSM with both higher sensitivity to social norms and self-efficacy were more likely to have HIV testing. This finding is consistent with previous literature that positive social norms and self-efficacy would work together in improving condom use among MSM [22]. There are several potential reasons for this phenomenon. First, MSM with higher sensitivity to social norms may be more likely to discuss HIV testing with friends [23]. This kind of interaction may further increase their self-efficacy and lead to HIV testing. Second, reasoned action theory has shown that when people are aware of their community's support for an act, they will be more likely to carry out such behavior [24]. Third, according to social cognitive theory, people with higher self-efficacy and sensitivity to social norms may make a greater effort to accomplish their goals in the near future [15]. Further implementation studies that can combine the social norms and self-efficacy strategies, and working together to improve HIV testing together would be more effective.

In our study, the prevalence of HIV testing among MSM remained low with $32.6 \%$ of participants having been tested for HIV in the last 3 months and $62.5 \%$ of participants having been tested for HIV in their lifetime. These testing proportions are higher than those from Thailand in 2014 [25] and Zhejiang, China in 2014 [1], but lower than those from Cambodia in 2015 [26]. The HIV testing proportion is still far behind the UNAIDS target for 90\% testing among infected individuals in 2014 [27]. This may be attributable to many factors. First, there is low awareness among MSM of the infection risk associated with sexual behavior [28]. Secondly, even MSM who are willing to be tested often need to travel to other cities for testing in order to avoid social stigma [29]. Thirdly, younger MSM are less likely to have been tested for HIV, potentially due to their fear of HIV testing in healthcare settings [30], in our study about $54.4 \%$ of the participants were 25 years old or younger. Lastly, the geographical distribution of HIV testing sites is unbalanced in China [31].

This study has several limitations. Firstly, we recruited participants through a mobile dating application, so participants tended to be younger, more highly educated, with a higher burden of syphilis and HIV [32]. MSM in remote rural areas in China were not included in the study. Secondly, HIV testing in the past 3 months was self-reported, which might lead to social desirability bias. Thirdly, the Cronbach's $\alpha$ of HIV testing social norm and self-efficacy were a bit low. Lastly, like many other online cross-sectional studies, there might be a selection bias in the study, as the online participants are tended to be young and well educated [33].

\section{Conclusions}

In summary, our results showed that the prevalence of HIV testing among MSM in China is suboptimal and may result in continuing transmission of HIV. However, most of the MSM who participated in this study were fairly confident in their likelihood of having HIV testing. Our study noted the association of the interaction between sensitivity to HIV testing-related social norms and self-efficacy with HIV testing and self-HIV testing among Chinese MSM. MSM with higher sensitivity to social norms and self-efficacy were more likely to have HIV testing. Future studies should investigate how social norms and self-efficacy are working together in promoting HIV testing. Policies and intervention packages (such as culturally competent sexual health education interventions [34]) should focus on increasing positive social norms and self-efficacy among MSM as an essential component of the overall intervention strategy.

\section{Additional file}

Additional file 1: Semiparametric regression map for social norm and self-efficacy. (TIFF $351 \mathrm{~kb}$ )

\begin{abstract}
Abbreviations
aOR: Adjusted odds ratio; cOR: Crude odds ratio; DAG: Directed acyclic graph; HIV: Human Immunodeficiency Virus; IQR: Interquartile range; MSM: Men who have sex with men; PITC: Provider-initiated testing and counseling; PLWH: People living with HIV; VCT: Voluntary counseling and testing
\end{abstract}

\section{Acknowledgments}

Thanks to Katherine Li for reviewing a prior version of this manuscript. This work is supported by the National Key Research and Development Program of China (2017YFE0103800), the National Institutes of Health [National Institute of Allergy and Infectious Diseases (NIAID) 1R01Al114310]; University of North Carolina (UNC)-South China STD Research Training Centre [Fogarty International Centre 1D43TW009532]; UNC Center for AIDS Research [NIAID 5P30AI050410]; and the Bill \& Melinda Gates Foundation to the Mesh Consortium (BMGF-OPP1120138); National Center for Advancing Translational Sciences [UL1TR001111] at the National Institutes of Health and Medical Scientific Research Foundation of Guangdong Province of China(A2018508). The listed grant funders played no role in any step of this study.

\section{Funding}

This work was supported by the National Key Research and Development Program of China (2017YFE0103800), the National Institutes of Health [National Institute of Allergy and Infectious Diseases 1R01Al114310]; UNCSouth China STD Research Training Centre [Fogarty International Centre 1D43TW009532]; UNC Center for AIDS Research [National Institute of Allergy and Infectious Diseases 5P30AI050410]; University of California San Francisco Center for AIDS Research [National Institute of Allergy and Infectious Diseases P30 Al027763]; and the Bill \& Melinda Gates Foundation to the Mesh Consortium (BMGF-OPP1120138. This publication was also supported by Grant Number UL1TR001111 from the National Center for Advancing Translational Sciences (NCATS) at the National Institutes of Health. Medical Scientific Research Foundation of Guangdong Province of China(A2018508). The listed grant funders played no role in any step of this study.

\section{Availability of data and materials}

The datasets used and/or analyzed during the current study are available from the corresponding author on reasonable request. 


\section{Authors' contributions}

$P Z, W T$, and $L L$ participated in all stages and wrote the manuscript, $B C, C L$, CW, and YZ helped collect the data; BY, CW, HC, WT, and JT helped design the study and reviewed the manuscript. All authors read and approved the final manuscript.

\section{Ethics approval and consent to participate}

Ethics review committees in China (Guangdong Provincial Center for Skin Diseases and STI Control) and the United States (University of North Carolina at Chapel Hill and the University of California, San Francisco) approved the study prior to launch. All participants agreed to informed consent and signed the inform consent prior to the survey. All the participants are Chinese, and they resided in China, and this study did get ethical approval from a Chinese IRB committee.

\section{Consent for publication}

Not applicable.

\section{Competing interests}

The authors declare that they have no competing interests.

\section{Publisher's Note}

Springer Nature remains neutral with regard to jurisdictional claims in published maps and institutional affiliations.

\section{Author details \\ 'Dermatology Hospital, Southern Medical University, Guangzhou, China ${ }^{2}$ Nanjing Municipal Center for Disease Control and Prevention, Jiangsu, China. ${ }^{3}$ The Third Affiliated Hospital, Sun Yat-Sen University, Guangzhou, China. ${ }^{4}$ SESH study group of University of North Carolina at Chapel Hill, Guangzhou, China. ${ }^{5}$ University of North Carolina at Chapel Hill Project-China, Guangzhou 510095, China. ${ }^{6}$ Social and Behavioral Health Sciences, School of Public Health, Rutgers University, Piscataway, NJ, USA. 'School of Medicine of University of North Carolina at Chapel Hill, Chapel Hill, USA.}

Received: 5 March 2018 Accepted: 23 October 2018

Published online: 30 October 2018

\section{References}

1. Li R, Pan X, Ma Q, Wang H, He L, Jiang T, Wang D, Zhang Y, Zhang X, Xia S Prevalence of prior HIV testing and associated factors among MSM in Zhejiang Province, China: a cross-sectional study. BMC Public Health. 2016;16(1):1152.

2. Lui C, Dean J, Mutch A, Mao L, Debattista J, Lemoire J, Howard C, Whittaker A, Hollingdrake O, Fitzgerald L. HIV testing in men who have sex with men: a follow-up review of the qualitative literature since 2010. AIDS Behav. 2018; 22(2):593-605.

3. Tucker JD, Wong FY, Nehl EJ, Zhang F. HIV testing and care systems focused on sexually transmitted HIV in China. Sex Transm Infect. 2012;1;88(2):116-9.

4. Zou H, Hu N, Xin Q, Beck J. HIV testing among men who have sex with men in China: a systematic review and meta-analysis. AIDS Behav. 2012;16(7):1717-28.

5. Evangeli M, Pady K, Wroe AL. Which psychological factors are related to HIV testing? A quantitative systematic review of global studies. AIDS Behav. 2016;20(4):880-918.

6. Chow $E$, Wilson $D$, Zhang $L$. The rate of HIV testing is increasing among men who have sex with men in China. HIV MED. 2012;13(5):255-63.

7. Perkins JM, Nyakato VN, Kakuhikire B, Mbabazi PK, Perkins HW, Tsai AC, Subramanian SV, Christakis NA, Bangsberg DR. Actual versus perceived HIV testing norms, and personal HIV testing uptake: a cross-sectional, population-based study in rural Uganda. AIDS Behav. 2018;22(2):616-28.

8. Prati G, Breveglieri M, Lelleri R, Furegato M, Gios L, Pietrantoni L. Psychosocial correlates of HIV testing among men who have sex with men in Italy: a cross-sectional study. Int J STD AIDS. 2013;25(7):496-503.

9. Babalola S. Readiness for HIV testing among young people in northern Nigeria: the roles of social norm and perceived stigma. AIDS Behav. 2007;11(5):759-69.

10. Safiri S. Knowledge, attitude, self-efficacy and estimation of frequency of condom use among Iranian students based on a crosswise model: more explanation is needed for the crosswise model. Int J Adolesc Med Health. 2017;29(2):20160110

11. Ahmed A, Weatherburn P, Reid D, Hickson F, Torres-Rueda S, Steinberg P, Bourne A. Social norms related to combining drugs and sex ("chemsex") among gay men in South London. Int J Drug Policy. 2016;38:29-35.
12. Jamil MS, Guy RJ, Bavinton BR, Fairley CK, Grulich AE, Holt M, Smith KS, Chen M, McNulty AM, Conway DP, et al. HIV testing self-efficacy is associated with higher HIV testing frequency and perceived likelihood to self-test among gay and bisexual men. Sex Health. 2017;14(2):170-8.

13. WHO: Consolidated guidelines on HIV testing services. 2015.

14. Jin SS, Bu K, Chen FF, Xu HF, Li Y, Zhao DH, Xu F, Li JY, Han MJ, Wang N, et al. Correlates of condom-use self-efficacy on the EPPM-based integrated model among Chinese college students. Biomed Environ Sci. 2017;30(2):97-105.

15. Ritchwood TD, Penn D, Peasant C, Albritton T, Corbie-Smith G. Condom use self-efficacy among younger rural adolescents:the influence of parent-teen communication, and knowledge of and attitudes toward condoms. J Early Adolesc. 2017;37(2):267-83.

16. Latkin CA, Kuramoto SJ, Davey-Rothwell MA, Tobin KE. Social norms, social networks, and HIV risk behavior among injection drug users. AIDS Behav. 2010:14(5):1159-68.

17. Rothman K, Greenland S, Lash TL. Modern. Epidemiology. 2008.

18. Khumsaen N, Stephenson R. Beliefs and perception about HIV/AIDS, selfefficacy, and HIV sexual risk behaviors among young Thai men who have sex with men. AIDS Educ Prev. 2017;29(2):175-90.

19. Zhou G, Li X, Qiao S, Zhou Y, Shen Z. Psychological and behavioral barriers to ART adherence among PLWH in China: role of self-efficacy. AIDS Care. 2017:29(12):1533-7.

20. Sun L, Yang SM, Wu H, Chen B, Wang CJ, Li XF. Reliability and validity of the Chinese version of the HIV treatment adherence self-efficacy scale in mainland China. Int J STD AIDS. 2017;28(8):829-37.

21. Guerra-Ordonez JA, Benavides-Torres RA, Onofre-Rodriguez DJ, MarquezVega MA, Guerra-Rodriguez GM, Wall KM. Self-efficacy and coping as correlates of migrant safe sexual behavior to prevent HIV. J Assoc Nurses AIDS Care. 2017;28(5):761-9.

22. Leddy A, Chakravarty D, Dladla S, de Bruyn G, Darbes L. Sexual communication self-efficacy, hegemonic masculine norms and condom use among heterosexual couples in South Africa. AIDS Care. 2015;28(2):228-33.

23. Zhou Q, Wu Y, Hong YA, Yang C, Cai W, Zhu Y, Guo Z, Guo Y. Association between perceived social norm and condom use among people living with HIV/AIDS in Guangzhou, China. AIDS Care. 2017:29(1):91-7.

24. Song F, Yan-ming S, Hong-yan LU, Xiao-yan MA, Hai Y, Li LU, Xiong HE, Wei $M A$, Xue-feng $L I$, Yan $X$, et al. Analysis on the relationship between condom social norms and unprotected anal intercourse among men who have sex with men in Beijing. Chinese J Epidemiol. 2011;32(5):473-6.

25. Sapsirisavat V, Phanuphak N, Keadpudsa S, Egan JE, Pussadee K, Klaytong P, Reuel Friedman M, van Griensven F, Stall R. Psychosocial and behavioral characteristics of high-risk men who have sex with men (MSM) of unknown HIV positive Serostatus in Bangkok, Thailand. AIDS Behav. 2016;20(S3):386-97.

26. Yi S, Tuot S, Chhoun P, Brody C, Pal K, Oum S. Factors associated with recent HIV testing among high-risk men who have sex with men: a crosssectional study in Cambodia. BMC Public Health. 2015;15(1).

27. UNAIDS. 90-90-90 - an ambitious treatment target to help end the AIDS epidemic. Switzerland: UNAIDS; 2014.

28. Cao B, Liu C, Durvasula M, Tang W, Pan S, Saffer AJ, Wei C, Tucker JD. Social media engagement and HIV testing among men who have sex with men in China: a Nationwide cross-sectional survey. J Med Internet Res. 2017;19(7):e251.

29. Zou H, Zhang L, Chow EPF, Tang W, Wang Z. Testing for HIV/STIs in China: challenges, opportunities, and innovations. Biomed Res Int. 2017;2017:1-3.

30. Wong NS, Tang W, Han L, Best J, Zhang Y. MSM HIV testing following an online testing intervention in China. 2017.

31. Chen W, Zhou F, Hall BJ, Tucker JD, Latkin C, Renzaho AMN, Ling L. Is there a relationship between geographic distance and uptake of HIV testing services? A representative population-based study of Chinese adults in Guangzhou, China. PLOS ONE. 2017;12(7):e180801.

32. Tang W, Best J, Zhang Y, Liu F, Tso LS, Huang S, Yang B, Wei C, Tucker JD. Gay mobile apps and the evolving virtual risk environment: a cross-sectional online survey among men who have sex with men in China. Sex Transm Infect. 2016;92(7):508-14.

33. Zhao P, Tang S, Wang C, Zhang Y, Best J, Tangthanasup TM, Huang S, Yang $B$, Wei C, Tucker JD, et al. Recreational drug use among Chinese MSM and transgender individuals: results from a National Online Cross-Sectional Study. PLoS One. 2017;12(1):e170024.

34. Cianelli R, Villegas N, McCabe BE, de Tantillo L, Peragallo N. Self-efficacy for HIV prevention among refugee Hispanic women in South Florida. J Immigr Minor Health. 2017;19(4):905-12. 